\title{
Evaluation of myocardial perfiusion and viability in coronary artery disease in view of the new revascularization
} guidelines

\section{Jolanta Miśko \\ Department of Magnetic Resonance, Institute of Cardiology, Warsaw, Poland}

\section{[Received 26 | 2012; Accepted 16 || 2012]}

of myocardial perfusion and viability assessments in the qualification of patients with coronary artery disease for further therapeutic management.

Based on these guidelines, myocardial perfusion assessment complete with the diagnosis of the extent and location of perfusion disorders provides the basis for the selection of patients with stable coronary artery disease or intermediate probability of coronary artery disease and normal resting contractility of the left ventricle for further invasive diagnostic (coronary angiography) and treatment (revascularisation) procedures [2].

And also, based on the same guidelines, myocardial viability assessment focused on the region supplied by the narrowed/occluded coronary artery forms the basis for the selection of patients with impaired contractility of the left ventricle for revascularisation $[3,4]$.

It is important that both these cases are relevant to stable patients or patients with intermediate probability of coronary artery disease for whom the postponement of therapeutic procedure by attempting preliminary non-invasive diagnostic methods should not cause any irreversible myocardial changes (necrosis) or any other major adverse cardiac events (MACE) [2]. Patients diagnosed using non-invasive methods for perfusion and viability assessment are those for whom it is possible - under no time pressure - 'cito' - to consider the benefits and drawbacks of the selected diagnostic and treatment procedures, and to prepare an appropriate management plan based on the clinical data and test results of a given patient. As has been stressed by the authors of the current guidelines concerning the indications for revascularisation, many patients should rather be considered eligible for planned coronary artery bypass grafting (CABG) than for emergency percutaneous transluminal coronary angioplasty (PTCA).

Individualisation of therapeutic procedures has now become the order of the day as a result of many years of experience and efficacy assessment of revascularisation versus optimal pharmacological therapy. 


\section{Myocardial perfusion}

Stable coronary artery disease causes insufficient blood supply to the myocardium during physical exertion (or under stress) and thus restricts patient's physical performance — the impairment of the coronary flow reserve is indicated under stress by such symptoms as coronary pain or its equivalent. The lack of these symptoms referred to as "silent ischemia" poses a danger for patients - it does not restrict their activity, does not trigger any danger signal and therefore can cause irreversible myocardial changes (necrosis). Patients with stable coronary artery disease can have normal resting myocardial contractility, which does not exclude stress-induced impairment of myocardial contractile function during physical exertion or immediately thereafter (hibernating and/or stunned myocardium). In the COURAGE study, in a subset of 300 patients with significant coronary stenosis who underwent revascularisation, the reduced risk of a major adverse cardiac event (MACE) was only reported for those patients who were observed to have an area of more than $10 \%$ of left ventricle affected with stress-induced blood supply disorders before the procedure - only those patients benefited from invasive treatment compared to optimal medical therapy [5].

Therefore a well-planned revascularisation procedure should aim at eliminating ischemia, and not only the coronary artery stenosis [6].

The best-known and most widely used methods for determining the location and extent of ischemia include perfusion scintigraphy (SPECT, single photon emission computed tomography) and echocardiography with pharmacological stress. Echocardiography with dobutamine stress allows doctors to assess the effects of ischemia, i.e. myocardial dysfunction. Such methods as single photon emission computed tomography (SPECT), positron emission tomography (PET) and cardiac magnetic resonance (CMR) enable direct assessment of myocardial perfusion. PET and CMR are less available than SPECT, but the prospect of their development and popularisation gives an opportunity of full quantitative assessment of myocardial blood flow (in $\mathrm{mL} / \mathrm{g} / \mathrm{min}$ ) and coronary flow reserve.

\section{Myocardial viability}

The diagnosis of potentially irreversible myocardial dysfunction is important on account of the higher risk of death in patients with coronary artery disease and reduced myocardial contractility [7]. Only $57 \%$ of the patients suffering from coronary artery disease with left ventricular ejection fraction below $35 \%$ can survive 4 years compared to the $92 \%$ survival rate among patients with ejection fraction over $50 \%[3,8]$. Post-revascularisation myocardial damage is potentially reversible in about $25-40 \%$ of the patients with coronary artery disease and LV dysfunction [9]. The meta-analysis of 3088 patients with coronary artery disease demonstrated that the death rate was 4 times lower among post-surgical patients with dysfunctional, but viable myocardium compared to patients on pharmacological treatment [3]. No significant difference was determined between the efficacy of pharmacological and surgical treatments among patients with no features of viable myocardium [3]. At the same time, one should be aware that the risk of death in the perioperative period and within a year from the coronary artery bypass graft (CABG) surgery in patients with permanent myocardial damage is twice as high compared to post-surgical patients with dysfunctional myocardium and retained myocardial viability (10\% and $7.7 \%$ vs $4 \%$ and $3.2 \%$ respectively). About $35 \%$ of CABG or PTCA patients do not benefit from the treatment due to the fact that revascularisation comprises the region of transmural myocardial necrosis [3].

Therefore the differentiation between reversible and irreversible myocardial damage enables prompt and appropriate qualification of patients for revascularisation, i.e.:

- selecting patients with potentially reversible damage who could benefit from surgical CABG or PTCA procedure;

- selecting patients with irreversible damage who should avoid non-beneficial surgical procedures or operations which might only increase the risk of complications and death.

However, the results from the earlier reports and the recommendations of the European Society of Cardiology (ESC) and the European Association for Cardio-Thoracic Surgery (EACTS) from 2010 are at variance with the results of the multicentre STICH study conducted in the years 2002-2007 among 2136 patients with ischemic myocardial dysfunction [10]. In the study, about half of the patients underwent SPECT or ECHO with dobutamine stress and they were not observed to derive greater benefits from surgical treatment compared to optimal medical therapy as a result of the retained myocardial viability. Ever since the publication of the STICH results in 2010, it has been discussed in the cardiological environment whether and how the limitations of the methodology selected for the study (PET or CMR instead of SPECT) could have affected the study results [11].

The diagnostic methods used for viability assessment are the same as those underlying perfusion assessment: echocardiography with dobutamine stress, perfusion scintigraphy, PET, MRI. Perfusion and viability assessments involve different study protocols and PET markers.

\section{Non-invasive imaging methods for myocardial perfusion and viability assessment}

\section{SPECT-based myocardial perfusion and viability assessment SPECT-BASED PERFUSION ASSESSMENT}

Myocardial perfusion and viability assessment is based on myocardial perfusion scintigraphy, currently applied as a computer-tomography method - single photon emission tomography (SPECT). Technetium-99m methoxyisobutylisonitrile (Tc-99m-MI$\mathrm{BI}$ ), an isonitrile derivative most often used as a SPECT imaging agent, enables identifying the level of perfusion and also marking non-damaged myocyte cell membrane [12-15].

SPECT-based perfusion assessment comprises two phases: exercise (or pharmacological stress) phase and resting phase. The comparison of the distribution of the (perfusion) marker accumulation in the myocardium in each phase enables the assessment of transient ischemia (i.e. reduced coronary flow reserve) (Figure 1) and permanent ischemia (i.e. myocardial necrosis - for artefact differentiation) (Figure 2). In the scintigraphic perfusion assessment it is assumed that at least one of the three regions of the coronary blood flow is properly supplied 


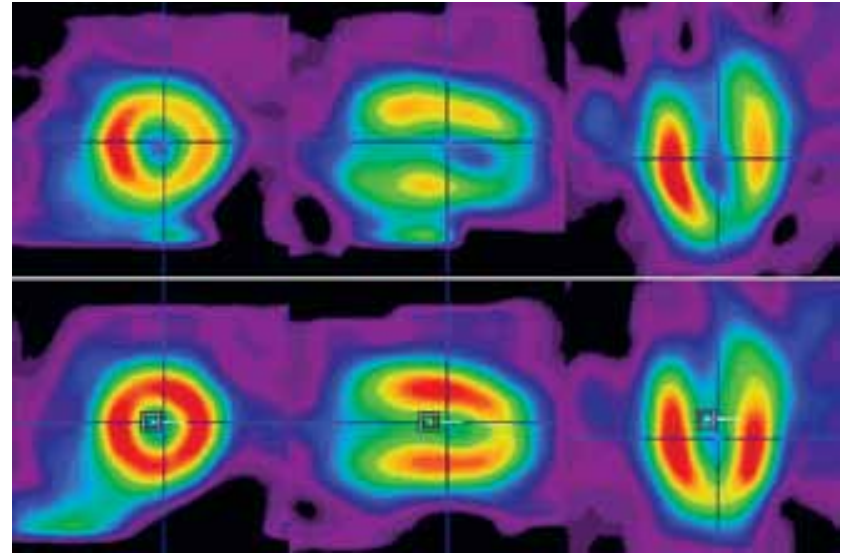

Figure 1. Myocardial perfusion SPECT study: stress (higher row) and rest (lower row) in short axis and also two-chamber and four- chamber long axis view. Reversible perfusion defects in anterior, apical, lateral and inferior myocardial walls are detected - decreased coronary flow reserve in more than $20 \%$ of left ventricle myocardium

with blood at rest and after stress — this reference region is necessary to assess improper perfusion in other regions. Hence the method is referred to as semi-quantitative and may be unreliable in patients with multivessel coronary disease. The sensitivity and specificity of the SPECT procedure in the assessment of myocardial ischemia is $85-90 \%$ and $70-75 \%$ respectively compared to coronary angiography results [2]. A region of improper perfusion larger than $10 \%$ of the left ventricle area [at least 2 segments in the 17-segment model (AHA)] is considered moderate to severe ischemia. Improper perfusion is most often defined as SD below 2.5 compared to the proper perfusion distribution data for healthy volunteers in the normative computer data base (commercially available software for semi-quantitative assessment: QGS/QPS - Cedars-Sinai, 4D-MSPECT, University of Michigan, Emory Toolbox - Emory University).

\section{SPECT-BASED VIABILITY ASSESSMENT}

Scintigraphic assessment of myocardial viability requires only resting SPECT image, and preferably resting ECG-gated SPECT (GSPECT) image.

It is the cheapest and most widely available viability imaging method among 99m-Tc-MIBI-based functional SPECT procedures - only a viable myocardium shows an appropriate level of perfusion at rest and systolic increase in the myocardial thickness during GSPECT registration [16,17]. During GSPECT acquisition a viable myocardium shows at least $50 \%$ of the maximal marker accumulation compared to the reference best blood-supplied myocardial region (assuming that it is non-damaged myocardium) and systolic increase in the myocardial thickness of at least $10 \%$ in ECG-gated SPECT registration. These SPECT parameters show that the myocardial functions are likely to improve in more than $70 \%$ of post-revascularisation patients [17]. In other studies, the level of marker accumulation during SPECT registration below 30\% of the maximal reference value shows that there is an $80 \%$ probability of myocardial non-viability, i.e. transmural or nearly transmural necrosis [14]. However, as shown in multicentre meta-analyses, SPECT-based viability values are overestimated and their specificity is only $49 \%$ with sensitivity at $88 \%$ [18].
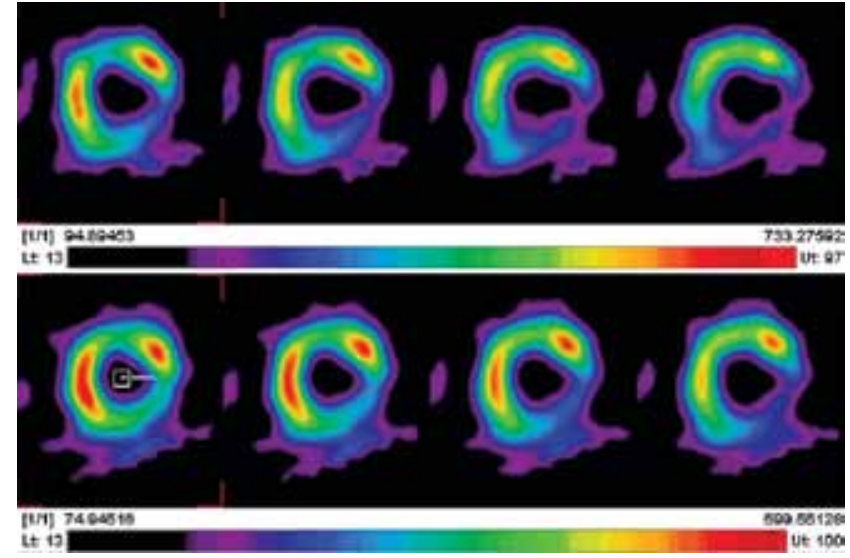

Figure 2. Myocardial perfusion SPECT study: stress (higher row) and rest (lower row) in short axis views. Persistent perfusion defect of infero-lateral wall at stress and rest intensifying at stress depicts subepicardial necrosis with still viable myocardium "at risk" in subepicardial part of these part of the wall

\section{PET-based myocardial perfusion and viability assessment \\ PET-BASED PERFUSION ASSESSMENT}

$P E T$ is considered a "gold standard" in myocardial perfusion assessment — the sensitivity and specificity of this method in the assessment of perfusion disorders in patients with coronary artery disease have been determined in 8 independent studies at 93-97\% respectively — on average $93 \%$ and $88-100 \%$ — on average $92 \%$ [19], and furthermore it enables quantitative assessment of perfusion in $\mathrm{mL} / \mathrm{g}$ of myocardial tissue/min using $\mathrm{N}-13 \mathrm{NH}_{3}$ (nitrogen-13 ammonia), $\mathrm{O}-15 \mathrm{H}_{2} \mathrm{O}$ (oxygen-15 water) or $\mathrm{Rb}-82 \mathrm{Cl}$ (rubidium-82 chloride). However, these markers have a short half-life and except for $\mathrm{Rb}-82$ need to be cyclotron-produced isotopes, and thus their cost is high. Nevertheless, only a fully quantitative assessment of coronary flow reserve and of coronary perfusion in $\mathrm{mL} / \mathrm{g} / \mathrm{min}$ during exercise and at rest can provide information whether and to what extent a patient with multivessel coronary disease suffers from impaired CFR of the myocardium supplied with blood through a narrowed coronary artery.

Greater coronary flow under stress is referred to as the coronary flow reserve (CFR) and measured as the ratio of the maximal blood flow to the blood flow at rest: coronary flow under stress/ /coronary flow at rest $=$ CFR.

Under normal conditions, CFR exceeds 2 which means that the increased blood flow in a coronary vessel under stress is at least twice as high as at rest.

If a sclerotic change in a coronary artery causes significant hemodynamic stenosis, then CFR decreases below 2 and reduced perfusion is observed in the myocardial region supplied by such coronary artery. Reduced CFR plays an important role in the development of indications for revascularisation $[1,20]$, unless it exceeds $10 \%$ of the myocardial area in accordance with the ESC guidelines.

\section{PET-BASED VIABILITY ASSESSMENT}

Fluorodeoxyglucose PET (FDG-PET) has recently been considered a "gold standard" in myocardial viability assessment [21], although currently this role has been taken over by MRI. In 
a viable myocardium with reduced blood supply, glucose is used as a source of energy in anaerobic metabolism. An increased accumulation of F-18-labelled glucose in the dysfunctional region is indicative of retained myocardial viability and contributes to the greater probability of improved left-ventricular efficiency after revascularisation $[22,23]$. There are two criteria relevant to PET-based myocardial viability assessment: 1) properly supplied with blood and viable, but dysfunctional myocardium, i.e. proper perfusion with normal or increased FDG accumulation indicates stunned myocardium, 2) improperly supplied with blood and viable, but dysfunctional myocardium, i.e. reduced perfusion with normal or increased FDG accumulation indicates a strong probability of hibernated myocardium [24]

However, PET is a costly procedure and has lower spatial resolution than $\mathrm{MRI}$ with strong enhancement after gadolinium administration. The comparison of PET and MRI results showed 95\% compatibility [25].

\section{CMR-based myocardial perfusion and viability assessment CMR-BASED PERFUSION ASSESSMENT}

The first pass of paramagnetic contrast agent through myocardium, i.e. so-called 'first-pass CMR' is performed twice: under pharmacological stress (dipyridamole, adenosine) and at rest [26]. The aim of the procedure is to assess the enhancement of signal intensity during the first pass of contrast agent through myocardium. The paramagnetic contrast agent reduces the relaxation time T1, i.e. the greater amount of the contrast agent flows into the coronary microcirculation with arterial blood, the higher is the signal intensity in a given LV myocardial segment. The CMR sequence used for registering perfusion 'first-pass' is Gradient Echo (GRE) - this very fast registration mode enables acquiring three short-axis images (basal, medial and apical) during one cardiac cycle. The images are recorded during subsequent phases of the cardiac cycle which enables dynamic registration of how the paramagnetic contrast agent flows through the cardiac cavities, and then to the coronary arteries and microcirculation with greater signal intensity in properly perfused regions of the myocardium. Signal intensity for the normal myocardium grows rapidly against time as opposed to the myocardium supplied by a narrowed coronary artery with delayed increase in signal intensity.

CMR procedure continues to improve; GRE sequence is modified in accordance with the technological development of MRI scanners, new paramagnetic contrast agents and new examination techniques are applied [27]. Currently, visual interpretation of perfusion images, including an assessment of the so-called signal intensity index during the first-pass CMR, plays a predominant role in everyday clinical practice. In the visual assessment it is assumed that at least one of the three regions of coronary blood supply shows normal perfusion at rest and normal coronary flow reserve under stress. This region is considered a reference region and the increase in its signal intensity is compared to the increase in signal intensity for the other myocardial segments. The result of the visual CMR-based perfusion assessment can be falsely negative in 3-vessel coronary artery disease, similarly as in the isotopic assessment of myocardial perfusion. Although there is a wide variety of computer programmes for semi-quantitative CMR-based perfusion assessment, no consensus have been reached as to which one could replace visual assessment. The semi-quantitative CMR-based perfusion assessment still requires a reference region of properly perfused myocardium (similarly as in SPECT), but the measurement of the intensity of perfusion disorders is numerical, and not subjective. Based on many years of experience, a range of different semi-quantitative parameters have been developed for the dynamic assessment of perfusion based on CT, SPECT and MRI examinations of organs of less mobility than heart (brain, kidneys, liver): upslope of the time-signal intensity curve during the inflow of the contrast agent, area under the time-signal intensity curve, maximal signal intensity within a given time interval, time needed to achieve the maximal signal intensity within a given myocardial region.

Although there is a theoretical basis for a fully quantitative CMR-based perfusion assessment, new papers are continued to be published and discussions are ongoing on the optimal acquisition protocol, dosage and rate of contrast agent administration as well as a post-processing programme which could enable precise and repeatable CFR assessment [28]. If a method for CMR-based perfusion procedure with quantitative perfusion assessment is developed, then such procedure will have a significant advantage over the quantitative PET-based assessment in multivessel coronary disease on account of the greater availability, lower price and lack of ionising radiation.

\section{CMR-BASED VIABILITY ASSESSMENT}

Over the past few years a number of publications [29-31] included reports on CMR-based myocardial necrosis imaging after the administration of paramagnetic contrast agent and with the signal ratio for viable and non-viable myocardium of up to $500 \%$. This method known as 'delayed enhancement' (DE) or 'late gadolinium enhancement' (LGE) has been successfully used for determining the presence, location and extent of both acute and chronic myocardial necrosis. The location, shape and transmural extent of delayed enhancement in the myocardium during DE MR examination is morphologically identical to the extent of necrosis determined in tetrazolium-based histopathological examination [32]. Increased Gd-DTPA accumulation overlaps with the histological cell changes in the necrotic region, typical of an infarct, and is about $130 \%$ higher than in a viable myocardium [33]. DE MR examination gives a possibility of determining the presence and extent of necrosis with spatial resolution greater than PET (gold standard thus far) or SPECT, and can be used to forecast improvement of myocardial functions after revascularisation (Figure 3).

Based on the results thus far, it was determined that the lack of delayed enhancement or the transmural extent of necrosis of up to $50 \%$ of the myocardial thickness increases the probability of post-revascularisation improvement of left-ventricular efficiency (Figure 4) [34]

\section{Summary}

Despite the existing discrepancies in the results of the usefulness studies concerning myocardial perfusion and viability assessments when qualifying patients with coronary artery disease for revascularisation, it is the prevailing view among cardiologists that any such information is relevant and new, less invasive imaging methods increase the comfort of making therapeutic decisions which are correct and beneficial for patients. 


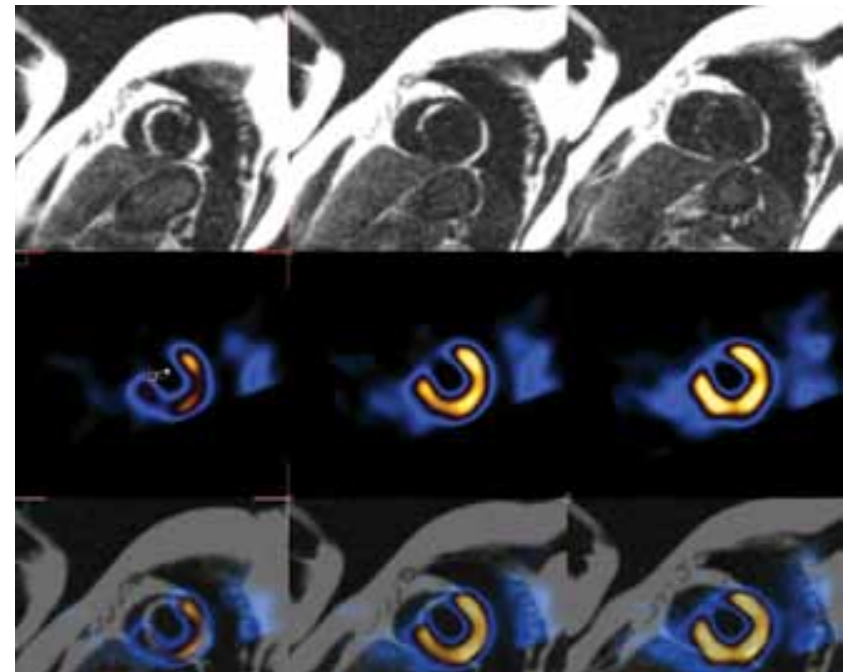

Figure 3. Comparison of delayed gadolinium enhancement magnetic resonance images (DE MR) and SPECT evaluation of transmural myocardial necrosis of antero-septal wall (without viability): delayed gadolinium enhancement MR (high row), SPECT with lack of tracer in area of MR contrast accumulation (middle row), image fusion of DE MR and SPECT images

Currently, the assessment of usefulness of non-invasive methods for perfusion and viability analyses based on the author's own experiences and available publications is given in the attached tables (Table 1, 2). The currently best results have been achieved for SPECT, including the cost of examination, its availability and objectivity. Similar requirements concerning myocardial viability assessment are satisfied by CMR. The classification presented

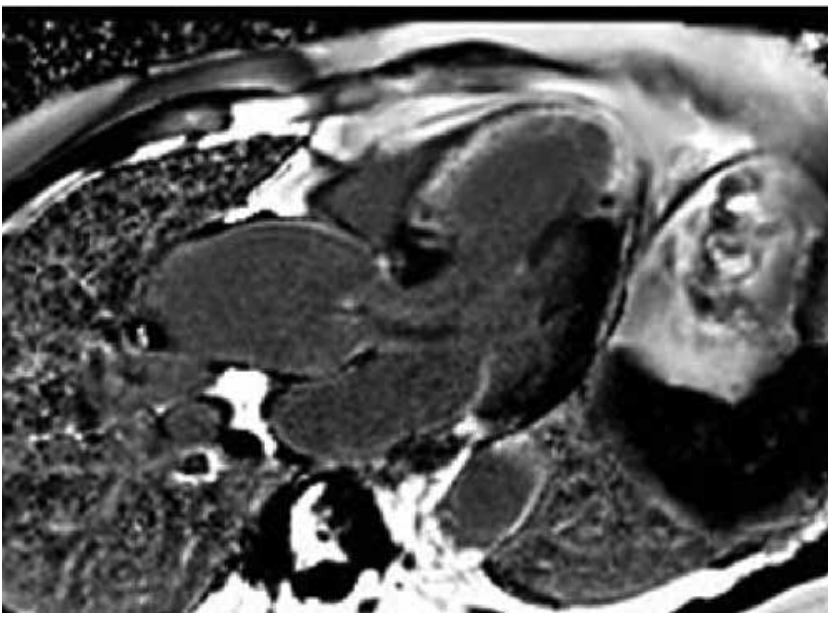

Figure 4. Nearly transmural necrosis of anteroseptal and apical walls on DE MR image in 3-chamber view - without signs of viability in these area

in the tables can change rapidly as it depends both on the technological progress, which is very dynamic in CMR, as well as the availability and costs of PET.

When analysing perfusion in patients with multivessel coronary disease, one should take into account the limitations of the SPECT and CMR methods - under such circumstances PET continues to be the 'gold standard' with quantitative assessment of perfusion and coronary flow reserve, where such assessment requires non-available or hardly available markers $\left(\mathrm{N}-13 \mathrm{NH}_{3}-\mathrm{ni}-\right.$ trogen-13 ammonia), $\mathrm{O}-15 \mathrm{H}_{2} \mathrm{O}$ - oxygen-15 water or $\mathrm{Rb}-82$-rubidium-82 chloride).

Table 1. Myocardial perfusion assessment using various imaging diagnostic methods

\begin{tabular}{|c|c|c|c|c|c|}
\hline Imaging method & Availability & $\begin{array}{c}\text { Cost } \\
\text { of examination }\end{array}$ & $\begin{array}{l}\text { Objective vs } \\
\text { subjective } \\
\text { assessment }\end{array}$ & $\begin{array}{c}\text { Semiquantitative/ } \\
\text { /quantitative } \\
\text { assessment }\end{array}$ & $\begin{array}{c}\text { Multivessel } \\
\text { disease }\end{array}$ \\
\hline SPECT & +++ & ++ & +++ & +++ & $+/++$ \\
\hline CMR & $++(?)$ & +++ & $++/+++$ & $+++(?)$ & $+(?)$ \\
\hline PET & $-(?)$ & ++++ & ++++ & ++++ & ++++ \\
\hline $\mathrm{ECHO}$ with dobutamine & +++ & + & $+/++$ & $+/++$ & $+/++$ \\
\hline
\end{tabular}

Myocardial perfusion assessment based on non-invasive imaging methods - additionally included echocardiography with dobutamine aimed at analysing functional disorders, i.e. indirectly the effects of perfusion disorders; - - non-available method/no diagnostic value/non-objective assessment; + - low availability/low diagnostic value/low cost/ /reduced objectivity; ++ - moderate availability/moderate diagnostic value/moderate cost/good objectivity; +++ - good availability/reliable and objective diagnostic value/ /moderate cost; ++++ - widely available method/high objectivity/high cost;? — developing/growing in popularity

Table 2. Myocardial perfusion assessment using various imaging diagnostic methods

\begin{tabular}{lccc}
\hline Imaging method & Availability & $\begin{array}{c}\text { Cost } \\
\text { of examination }\end{array}$ & $\begin{array}{c}\text { Objective vs } \\
\text { subjective assessment }\end{array}$ \\
\hline SPECT & +++ & ++ & $++/+++$ \\
quantitative assessment & +++++ \\
CMR & $++(?)$ & ++++ & +++++ \\
PET & $+(?)$ & + & ++++++++++++++++
\end{tabular}

Myocardial perfusion assessment based non-invasive imaging methods - additionally included echocardiography with dobutamine aimed at analysing the potential postrevascularisation functional improvement; - - non-available method/no diagnostic value/non-objective assessment; + - low availability/low diagnostic value/low cost/reduced objectivity; + + - moderate availability/moderate diagnostic value/moderate cost/moderate objectivity; +++- good availability/reliable and objective diagnostic value/low cost; ++++- widely available method/high objectivity/high cost;? — developing/growing in popularity 


\section{References}

1. Wijns W, Kolh P, Danchin N et al. Guidelines on myocardial revascularization The Task Force on Myocardial Revascularization of the European Society of Cardiology (ESC) and the European Association for Cardio-Thoracic Surgery (EACTS). Eur Heart J 2010; 31: 2501-2555.

2. Fox K, Garcia MA, Ardissino D et al. Guidelines on the management of stable angina pectoris: executive summary: the Task Force on the Management of Stable Angina Pectoris of the European Society of Cardiology. Eur Heart J 2006; 27: 1341-1381.

3. Allman KC, Shaw LJ, Hachamovitch R, Udelson JE. Myocardial viability testing and impact of revascularization on prognosis in patients with coronary artery disease and left ventricular dysfunction: a meta-analysis. J Am Coll Cardiol 2002; 39: 1151-1158.

4. Beanlands RS, Nichol G, Huszti E et al. F-18-fluorodeoxyglucose positron emission tomography imaging-assisted management of patients with severe left ventricular dysfunction and suspected coronary disease: a randomized, controlled trial (PARR-2). J Am Coll Cardiol 2007; 50: 2002-2012.

5. Shaw LJ, Berman DS, Maron DJ et al. Optimal medical therapy with or without percutaneous coronary intervention to reduce ischemic burden: results from the Clinical Outcomes Utilizing Revascularization and Aggressive Drug Evaluation (COURAGE) trial nuclear substudy. Circulation 2008; 117: 1283-1291.

6. Davies RF, Goldberg AD, Forman S et al. Asymptomatic Cardiac Ischemia Pilot (ACIP) study two-year follow-up: outcomes of patients randomized to initial strategies of medical therapy versus revascularization. Circulation 1997; 95: 2037-2043.

7. Soto JR, Beller GA. Clinical benefit of noninvasive viability studies of patients with severe ischemic left ventricular dysfunction. Clin Cardiol $2001 ; 24:$ 428-434

8. Pasquet A, Robert A, D'Hondt AM, Dion R, Melin JA, Vanoverschelde $\mathrm{JL}$. Prognostic value of myocardial ischemia and viability in patients with chronic left ventricular ischemic dysfunction. Circulation 1999; 100: 141-148.

9. Bax JJ et al. 18-Fluorodeoxyglucose imaging with positron emission tomography and single photon emission computed tomography: cardiac applications. Semin Nucl Med 2000; 30: 281-298.

10. STICH (Surgical Treatment for Ischemic Heart Failure) trial enrollment. Jones RH, White H, Velazquez EJ et al. J Am Coll Cardiol 2010; 56: 490-498.

11. Szwed H. Comment to article: Czy już powinniśmy zmienić obowiązujący pogląd na temat oceny żywotności przed zabiegiem rewaskularyzacji? Kardiol Pol 2011; 69: 1097-1098.

12. Sciagra R, Sestini S, Bolognese L, Cerisano G, Buanamici P, Pupi A. Comparison of dobutamine echocardiography and 99mTc-Sestamibi tomography for prediction of left ventricular ejection fraction outcome after acute myocardial infarction treated with successful primary coronary angioplasty. J Nucl Med 2002; 43: 8-14.

13. Dilsizian V, Arrighi JA, Diodati JG et al. Myocardial viability in patients with chronic coronary artery disease — comparison of 99mTc-Sestamibi with Thallium reinjection and $18 \mathrm{~F}$ Fluorodeoxyglucose. Circulation 1994; 89: 578-587.

14. Cuocolo A, Acampa W, Nicolai E, Pace L, Petretta M, Salvatore M. Quantitative thallium-201 and technetium 99m sestamibi tomography at rest in detection myocardial viability in patients with chronic ischemic left ventricular dysfunction. J Nucl Cardiol 2000; 7: 8-15.

15. Klocke FJ, Baird MG, Lorell BH et al. ACC/AHA/ASNC guidelines for the clinical use of cardiac radionuclide imaging - executive summary: a report of the American College of Cardiology/American Heart Association task Force on Practise Guidelines (ACC/AHA/ASNC Committee to Revise the 1995 Guidelines for the Clinical Use of Cardiac Radionuclide Imaging) J Am Coll Cardiol 2003; 42: 1318-1333.
16. Chua T, Kiat H, Germano G, Maurer G, Train K, Friedman J, Berman D. Gated Technetium-99m Sestamibi for simultaneous assessment of stress myocardial perfusion, postexercise regional ventricular function and myocardial viability. J Am Coll Cardiol 1994; 23: 1107-1114.

17. Murashita T, Makino Y, Kamikubo Y, Yasuda K, Mabuchi M, Tamaki N.J Quantitative gated myocardial perfusion single photon emission computed tomography improves the prediction of regional functional recovery in akinetic areas after coronary bypass surgery: useful tool for evaluation of myocardial viability. Thorac Cardiovasc Surg 2003; 126: 1328-1334.

18. Bax JJ, Wijns W, Cornel JH et al. Accuracy of currently available techniques for prediction of functional recovery after revascularization in patients with left ventricular dysfunction due to chronic coronary artery disease: Comparison of pooled data. J Am Coll Cardiol 1997; 30: 1451-1460.

19. Berman DS, Germano G. Interpretation and reporting of gated myocardial perfusion SPECT. In: Germano G, Berman DS, eds. Clinical Gated Cardiac SPECT. 2nd ed. U.K. Blackwell Publishing, Oxford 2006.

20. Schwitter J, Nanz D, Kneifel S et al. Assessment of myocardial perfusion in coronary artery disease by magnetic resonance: a comparison with positron emission tomography and coronary angiography. Circulation 2001; 103: 2230-2234.

21. Eitzman D, al-Aouar Z, Kanter $\mathrm{HL}$ et al. Clinical outcome of patients with advanced coronary artery disease after viability studies with positron emission tomography. J Am Coll Cardiol 1992; 20: 559-565.

22. Di Carli MF, Davidson M, Little R et al. Value of metabolic imaging with positron emission tomography for evaluating prognosis in patients with coronary artery disease and left ventricular dysfunction. Am J Cardiol 1994; 73: 527-533.

23. Tamaki N, Kawamoto M, Tadamura E et al. Prediction of reversible ischemia after revascularization — perfusion and metabolic studies with positron emission tomography. Circulation 1995; 91: 1697-1705.

24. vom Dahl J, Herman WH, Hicks RJ et al. Myocardial glucose uptake in patients with insulin-dependent diabetes mellitus assessed quantitatively by dynamic positron emission tomography. Circulation 1993; 88: 395-404.

25. Kuhl HP, Beek AM, van der Weerdt AP et al. Myocardial viability in chronic ischemic heart disease: comparison of contrast-enhanced magnetic resonance imaging with (18)F-fluo-rodeoxyglucose positron emission tomography. J Am Coll Cardiol 2003; 41: 1341-1348.

26. Schwitter J. Myocardial perfusion. J Magn Reson Imaging 2006; 24 : 953-963.

27. Manning WJ, Atkinson DJ et al. First-pass nuclear magnetic resonance imaging studies using gadolinium-DTPA in patients with coronary artery disease. J Am Coll Cardiol 1991; 18: 959-965.

28. Jerosch-Herold M, Wilke N, Stillman AE. Med Phys 1998; 25: 73-84.

29. Choi KM, Kim RJ, Gubernikoff G, Vargas JD, Parker M, Judd RM. Transmural extent of acute myocardial infarction predicts long-term improvement in contractile function. Circulation 2001; 104: 1101-1107.

30. Kim RJ, Wu E, Rafael A et al. The use of contrast-enhanced magnetic resonance imaging to identify reversible myocardial dysfunction. N Engl J Med 2000; 343: 1445-1453.

31. Kim RJ, Judd RM. Fundamental concepts in myocardial viability assessment revisited: when knowing how much is "alive" is not enough. Heart 2004; 90: 137-140.

32. Fieno DS, Kim RJ, Chen EL, Lomansney JW, Klocke FJ, Judd RM: Contrast - enhanced magnetic resonance imaging of myocardium at risk: distinction between reversible and irreversible injury throughout infarct healing. J Am Coll Cardiol 2000; 36: 1985-1991.

33. Rehwald WG, Fieno DS, Chen EL, Kim RJ, Judd RM. Myocardial magnetic resonance imaging contrast agent concentration after reversible and irreversible ischemic injury. Circulation 2002; 105: 224-229.

34. Gerber BL, Garot J, Bluemke DA, Wu KC, Lima JAC. Accuracy of contrast-enhanced magnetic resonance imaging in predicting improvement of regional myocardial function in patients after acute myocardial infarction. Circulation 2002; 106: 1083-1089. 